\title{
Increased Serum Glucose is not a Covid-19 Mortality Predictor in Elderly Patients
}

\author{
Luis R Macias Kauffer ${ }^{1}$, Maura Robledo Cayetano', Mónica Sierra Martínez ${ }^{2}$, Bryan Medina \\ Leyte $^{1}$, Alma R Sánchez Conejo ${ }^{3}$ and Gustavo Acosta Altamirano ${ }^{1 *}$ \\ ${ }^{1}$ Research Directorate, Ixtapaluca Regional High Specialty Hospital, Mexico
}

${ }^{2}$ General Directorate, Regional Hospital of High Specialty of Ixtapaluca, Mexico

${ }^{3}$ General Management, Ixtapaluca Regional High Specialty Hospital, Mexico

*Corresponding author: Gustavo Acosta Altamirano, Directorate of Planning, Teaching and Research, Ixtapaluca Regional High Specialty Hospital, Mexico

To Cite This Article: Luis R Macias Kauffer, Maura Robledo Cayetano, Bryan Medina Leyte, Alma R Sánchez Conejo, Gustavo Acosta Altamirano, Increased Serum Glucose is not a Covid-19 Mortality Predictor in Elderly Patients. 2020 - 11(1). AJBSR.MS.ID.001613. DOI: 10.34297/ AJBSR.2020.11.001613.

Received: 眥 November 30, 2020; Published: 战 December 12, 2020

\begin{abstract}
COVID-19 is causing a current pandemic with high mortality, in which older age is an important risk factor. Few studies have examined whether risk factors for death by COVID-19 are similar in elderly and younger patients. We reviewed electronic hospital records of all consecutive adult patients admitted and analyzed reported COVID-19 mortality risk factors stratified by age group (under 60 vs. 60 and over). In univariate analyses admission date, sex, age, oxygen saturation, hyperglycemia (defined as admission glucose $>180 \mathrm{mg} / \mathrm{dl}$ ) and lactate dehydrogenase were associated with COVID-19 mortality in younger patients, while only sex, oxygen saturation and lactate dehydrogenase were associated with mortality in the elderly. Mortality for younger subjects without hyperglycemia was $39.1 \%$, while it was $66.7 \%$ in for younger subjects with hyperglycemia, similar to the mortality in the 60 and over age group (66.7\% non-hyperglycemic, $60.3 \%$ hyperglycemic). This pattern was also observed in multivariate logistic regression with COVID-19 mortality as outcome, where after adjusting for admission date, sex, age, oxygen saturation, lactic dehydrogenase and D-dimer the OR for hyperglycemia was 2.34 (95\% CI 1.11-5.03) in the younger age group, while in the elderly the 0R was 0.50 (95\% CI $0.17-1.37$ ), with evidence of statistical interaction ( $\mathrm{p}=0.025)$. This behavior could be explained by an acute effect of SARS-CoV-2 on pancreatic tissue that affects mainly younger subjects. In conclusion, hyperglycemia is not associated with increased mortality in subjects aged over 60 .
\end{abstract}

\section{Introduction}

The COVID-19 pandemic, declared by WHO on March 2020, is responsible for at least 1.4 million deaths [1], with roughly half of these deaths occurring in the WHO Region of The Americas. Public data released from Mexican health authorities disclose a casefatality ratio of $9.6 \%$, while case-fatality ratio in the United States of America is $2.1 \%$ and $2.8 \%$ in Brazil. Within Mexico, 21.9\% of deaths occurred in the Mexico City Metropolitan Area [2], this is consistent with the proportion of the population that lives in this territory. Early and recent reports have established age as an important risk factor for COVID-19 mortality [3,4]. An ageing population has been put forth as a cause for high COVID-19 mortality [5]. In Mexico however, people aged 65 and over are $7.2 \%$ of the population and although case fatality is lower in subjects under 60 it is still $4.6 \%$. Alternative explanations for a higher mortality in Mexican patients have been an obesity prevalence in adults of $33.3 \%$ accompanied by a diabetes prevalence of $13.7 \%$ [6].

On the other hand, some studies have shown that disease characteristics differ in elderly patients when compared to younger patients. A small study (56 patients) found similar symptoms, but higher C- reactive protein among elderly patients [7]. A slightly larger study also reports similar symptoms but a higher frequency of comorbidities, higher D-dimer and lactate dehydrogenase among elderly patients [8]. However, these studies did not analyze if these features were related with mortality by COVID-19. Finally, a study of 1,520 patients aged 65 and over that did not include younger patients found an association between low oxygen saturation and severe lymphopenia with hospital mortality [9]. To evaluate whether COVID-19 mortality risk factors affect elderly and young 
patients alike, we sought to describe the characteristics of elderly COVID-19 patients in a tertiary care setting that underwent extensive conversion in response to the epidemiological risk [10] and to evaluate the effect of previously reported COVID-19 mortality risk factors in elderly and young adult COVID-19 patients.

\section{Methods}

Electronic hospital records from consecutive patients admitted to the Ixtapaluca Regional High Specialty Hospital from March $13^{\text {th }}$ (date the first COVID-19 patient was received) through 31 July were reviewed. This is a public hospital that covers a mainly uninsured population from the eastern outskirts of Mexico City. Nasopharyngeal swabs were obtained from patients that attended the Emergency Department with 1 of the following: cough, shortness of breath, fever or headache; and an additional symptom which could consist in myalgia, arthralgia, sore throat, chills, thoracic pain, rhinorrhea or conjunctivitis. Subjects were included if reverse transcription PCR for SARS-CoV-2 was positive. Pregnant women and subjects under 18 years of age were excluded. Attending physicians and two of the authors (LM, MR) extracted selected information regarding age, sex, vital signs including oxygen saturation on admission, symptoms, comorbidities, laboratory results on admission and outcome (discharge vs death). Diabetes and hypertension were considered present if the patient manifested a previous diagnosis or glucose lowering or antihypertensive medication was used. respectively. Because approximately a third of diabetic patients in Mexico are undiagnosed [6] we also analyzed COVID-19 mortality risk with hyperglycemia. Since serum glucose on admission could be fasting or post-prandial we defined hyperglycemia as levels above $180 \mathrm{mg} / \mathrm{dl}$, the upper limit of acceptable glucose values in critical patients [11]. We divided subjects into those under 60 years of age and those 60 and older. Characteristics from each group is presented as median (interquartile range) or percentage (95\% confidence interval). Qualitative variables were compared with the $\chi 2$ test, while differences in quantitative variables were assessed with the Wilcox test. Age-group stratified association of potential risk factors with mortality was done using logistic regression both for univariate and multivariate tests. Homogeneity of odds ratio was evaluated with the Breslow-Day test. Risk factors evaluated were admission date, sex, age, overweight or obese status, history of diabetes, history of hypertension, oxygen saturation, hyperglycemia, lactate dehydrogenase and D-dimer. These factors were selected based on existing literature [7-9,12]. If any of these were associated with mortality at the $\alpha=0.05$ level they were included in multivariate analysis. Finally, we tested if the effects of hyperglycemia differed by age group with a non-stratified multinomial logistic regression that included an interaction term between these factors. All analyses were performed in R 4.0.3.

\section{Results}

We analyzed the outcome of 646 hospitalized adults with a COVID-19 molecular diagnosis. Median age was 53 years, with an interquartile range of 44-63 years. A total 334 (51.7\%) patients, died. When divided by age groups, 426 were aged under 60 and 220 were 60 and over. Both diabetes and hypertension were are more frequent in the 60 and over group. Fever was frequent in the 60 and over group (82.51\%), although slightly less so than in the under 60 group (88.4\%) and this difference did no attain statistical significance, while diarrhea was significantly less frequent in the 60 and over group. Regarding laboratory measurements, D dimer and glucose was higher, while hemoglobin and triglycerides were lower in the 60 and over group. Mortality was significantly higher in the 60 and over group (Table 1 ).

Table 1: Characteristics of SARS-CoV-2 rtPCR positive patients by age group.

\begin{tabular}{|c|c|c|c|}
\hline & Aged under $\mathbf{6 0} \mathbf{n}=\mathbf{4 2 6}$ & Aged $\mathbf{6 0}$ and over $\mathbf{n} \mathbf{2 2 0}$ & 0.0194 \\
\hline Female (\%) & $32.39(32.3-32.5)$ & $41.82(41.6-42)$ & 0.0043 \\
\hline Overweight (\%) & $38.05(37.9-38.2)$ & $38.96(38.7-39.3)$ & $<0.001$ \\
\hline Obese (\%) & $47.79(47.6-47.9)$ & $30.52(30.2-30.8)$ & $<0.001$ \\
\hline Diabetes (\%) & $24.41(24.3-24.5)$ & $40.91(40.7-41.1)$ & $<0.001$ \\
\hline Hypertension (\%) & $22.41(22.3-22.5)$ & $44.75(44.5-45)$ & 0.0643 \\
\hline Fever (\%) & $88.4(88.3-88.5)$ & $82.51(82.4-82.7)$ & 0.9234 \\
\hline Headache (\%) & $59.12(59-59.3)$ & $59.88(59.6-60.2)$ & 0.0115 \\
\hline Diarrhea (\%) & $11.66(11.6-11.7)$ & $4.49(4.4-4.5)$ & 0.3709 \\
\hline Shortness of breath (\%) & $85.03(85-85.1)$ & $88.14(88-88.2)$ & $75(60-84)$ \\
\hline O $_{2}$ saturation $(\%)$ & $80(68-87)$ & $9.95(7.9-14.4)$ & 0.001 \\
\hline Leucocytes $\left(\mathrm{X} 10^{3} / \mu \mathrm{l}\right)$ & $9.95(7.3-13.8)$ & $3(0.9-9)$ & 0.18 \\
\hline Lymphocytes $\left(\mathrm{X} 10^{3} / \mu \mathrm{l}\right)$ & $3(0.9-9)$ & $14.6(13.1-15.7)$ & 0.3574 \\
\hline Hemoglobin $(\mathrm{g} / \mathrm{dl})$ & $15.2(13.6-16.3)$ & $418(327.2-537)$ & 0.004 \\
\hline Lactic dehydrogenase $(\mathrm{ng} / \mathrm{ml})$ & $384(300-515)$ & 0.1966 \\
\hline
\end{tabular}




\begin{tabular}{|c|c|c|c|}
\hline D dimer $(\mathrm{ng} / \mathrm{ml})$ & $995.2(630-1900)$ & $1740(912.5-3656)$ & $<0.001$ \\
\hline Serum glucose $(\mathrm{mg} / \mathrm{dl})$ & $118(97-156.5)$ & $142(109-224)$ & 0.001 \\
\hline Serum cholesterol $(\mathrm{mg} / \mathrm{dl})$ & $126(106-151.5)$ & $123(103-142$ & 0.1232 \\
\hline Serum triglycerides $(\mathrm{mg} / \mathrm{dl})$ & $162(124-210)$ & $147(118-189)$ & 0.2085 \\
\hline Ferritin $(\mathrm{ng} / \mathrm{ml})$ & $742(369.9-1286.8)$ & $785.38(469-1440.2)$ & 0.7835 \\
\hline Mechanical ventilation $(\%)$ & $21.74(21.6-21.9)$ & $20.17(19.9-20.4)$ & $65.91(65.7-66.1)$ \\
\hline
\end{tabular}

Note: Values for qualitative variables are medians (interquantile range), values for quantitative variables are percentages (95\% confidence interval). Difference by age groups was assessed with the $\chi 2$ test for qualitative variables and with the Wilcox test for quantitative variables.

\section{Univariate Association with COVID-19 Mortality in Different Age Groups}

Concerning the relationship with timing within the COVID-19 epidemic, a decline in mortality was observed with later hospital admission in the under 60 age group (beta $=-0.11, p=0.003$ ), but not in the 60 and over group (beta $=-0.06, p=0.233$ ) (Figure 1 ). The association between COVID-19 mortality and reported risk factors is presented in Table 2. The odds ratio (OR) for sex and mortality, both in subjects under 60 as in the 60 and over group shows a protective effect in females, with an OR of 0.4 (95\% confidence interval [CI] 0.26-0.61) in the former, and of 0.49 (95\% CI 0.28 0.86 ) in the latter. The Breslow-Day test supports a difference in OR between the age groups, albeit having the same direction in both $(p=9.81 X 10-07)$. Age was associated with mortality in the under 60 age group (OR = 1.06 per additional year, 95\% CI 1.03-1.08), with a similar albeit non-significant behavior in the 60 and over group (OR $=1.03$ per additional year, 95\% CI 0.99-1.07). Weight and height measurements were available for 339 (79.58\%) under 60 patients and $154(70 \%)$ patients 60 and over. Within the younger subjects only $12.39 \%$ have a body mass index (BMI) between 20.0 y $24.9 \mathrm{~kg} /$ $\mathrm{m} 2$, i.e. within the normal range. Neither overweight or obesity were associated with mortality in either age group (under 60 OR
1.5 IC 95\% 0.79-2.93, 60 and over OR 0.79 IC 95\%0.37-1.61). The proportion of patients in the whole sample with a previous type 2 diabetes (T2D) diagnosis was $30.03 \%$. For the association between T2D and COVID-19 mortality we observe differing trends in each age group. The direction of the association between COVID-19 mortality and history of diabetes pointed towards risk in the under 60 age group ( $\mathrm{OR}=1.35$ ) while it was inclined to protection in those 60 and older (OR $=0.59$ ), although statistical significance was not reached in neither. When serum glucose on admission above $180 \mathrm{mg} / \mathrm{dl}$ was considered a similar pattern is observed, with significantly increased risk $(O R=3.1,95 \%$ CI 1.86-5.31) in subjects under 60 , while in the older patient group no association is observed although the point estimate is protective (OR $=0.76,95 \%$ CI 0.41-1.42). The Breslow-Day test suggests non-homogeneous ORs ( $p=4.9210 \mathrm{X} 10-6)$. The mortality of subjects under 60 with hyperglycemia is similar to that of subjects 60 and over (Figure 3). As was the case for T2D, an inverted effect on mortality depending on age group is observed for hypertension. However, this effect does not reach statistical significance in either age group. Oxygen saturation and lactate dehydrogenase on admission showed a strong association with COVID- 19 mortality in both age groups. D-dimer was only associated with COVID-19 mortality in the younger patients.

Table 2: Univariate associations with COVID-19 mortality in subjects aged under 60 and 60 and over.

\begin{tabular}{|c|c|c|c|c|c|c|}
\hline & \multicolumn{3}{|c|}{ Aged under 60} & \multicolumn{3}{|c|}{ Aged 60 and over } \\
\hline & OR & $95 \% \mathrm{CI}$ & $\mathrm{p}$ & OR & $95 \% \mathrm{CI}$ & $\mathrm{p}$ \\
\hline Admission date (10 day increase) & 0.90 & $0.83-0.96$ & $0.83-0.96$ & 0.94 & $0.84-1.04$ & 0.233 \\
\hline Female sex & 0.40 & $0.26-0.61$ & $<0.001$ & 0.49 & $0.28-0.86$ & 0.013 \\
\hline Age & 1.06 & $1.03-1.08$ & $<0.001$ & 1.03 & $0.99-1.07$ & 0.185 \\
\hline Overweight or obese & 1.50 & $0.79-2.93$ & 0.220 & 0.79 & $0.37-1.61$ & 0.515 \\
\hline Diabetes & 1.35 & $0.87-2.11$ & 0.184 & 0.59 & $0.34-1.04$ & 0.069 \\
\hline Hypertension & 1.54 & $0.97-2.44$ & 0.658 & 1.01 & $0.58-1.78$ & 0.974 \\
\hline Oxygen saturation & 0.94 & $0.93-0.96$ & $<0.001$ & 0.92 & $0.89-0.94$ & $<0.001$ \\
\hline Hyperglycemia & 3.11 & $1.86-5.31$ & $<0.001$ & 0.79 & $0.41-1.42$ & 0.383 \\
\hline Lactate dehydrogenase & 1.01 & $1-1.01$ & $<0.001$ & 1.01 & $1-1.01$ & $<0.001$ \\
\hline D-dimer & 1.00 & $1-1$ & 0.002 & 1.00 & $1-1$ & 0.621 \\
\hline
\end{tabular}

Note: Odds ratios and $95 \%$ confidence intervals were calculated with logistic regression with mortality as the outcome of interest. 
Table 3: Multivariate associations with COVID-19 mortality in subjects aged under 60 and 60 and over.

\begin{tabular}{|c|c|c|c|c|}
\hline \multirow{8}{*}{ Under 60} & & OR & $95 \% \mathrm{CI}$ & $\mathbf{p}$ \\
\hline & Admission date (10 day increase) & 0.84 & $0.75-0.94$ & 0.002 \\
\hline & Female sex & 0.64 & $0.33-1.21$ & 0.152 \\
\hline & Age (year increase) & 1.03 & $1.00-1.07$ & 0.0434 \\
\hline & Oxygen saturation (\% increase) & 0.97 & $0.95-0.99$ & 0.016 \\
\hline & Hyperglycemia & 2.34 & $1.11-5.03$ & 0.027 \\
\hline & Lactic dehydrogenase & 1 & $1.00-1.01$ & $<0.001$ \\
\hline & D-dimer & 1 & $1.00-1.00$ & 0.166 \\
\hline \multirow{9}{*}{60 and over } & & OR & $95 \% \mathrm{CI}$ & $\mathbf{p}$ \\
\hline & Admission date & 1.04 & $0.87-1.26$ & 0.641 \\
\hline & (10 day increase) & & & \\
\hline & Female sex & 0.49 & $0.18-1.26$ & 0.141 \\
\hline & Age (year increase) & 1.07 & $1.00-1.16$ & 0.048 \\
\hline & Oxygen saturation (\% increase) & 0.92 & $0.87-0.96$ & $<0.001$ \\
\hline & Hyperglycemia & 0.5 & $0.17-1.37$ & 0.182 \\
\hline & Lactic dehydrogenase & 1.01 & $1.00-1.01$ & 0.006 \\
\hline & D-dimer & 1 & $1.00-1.00$ & 0.304 \\
\hline
\end{tabular}

Note: Odds ratios and $95 \%$ confidence intervals were calculated with logistic regression with mortality as the outcome of interest.

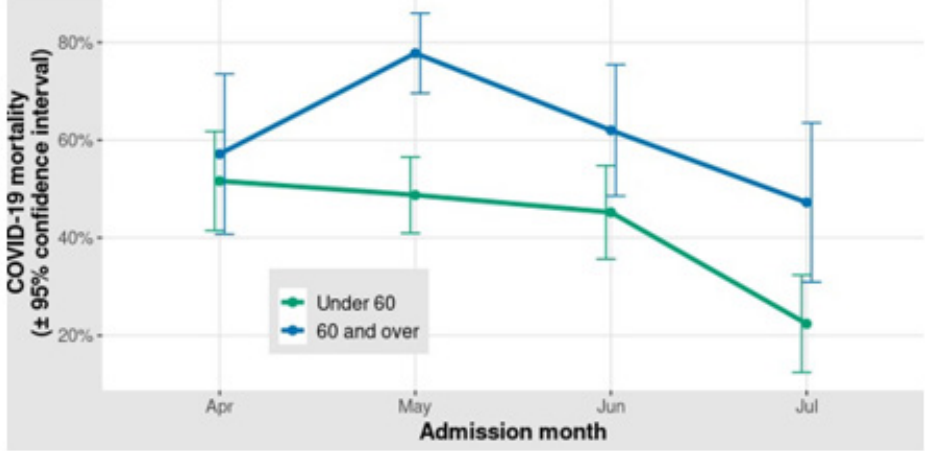

Figure 1: COVID-19 mortality according to admission month and age group. Confidence intervals for each of the time points was calculated assuming a binomial distribution.

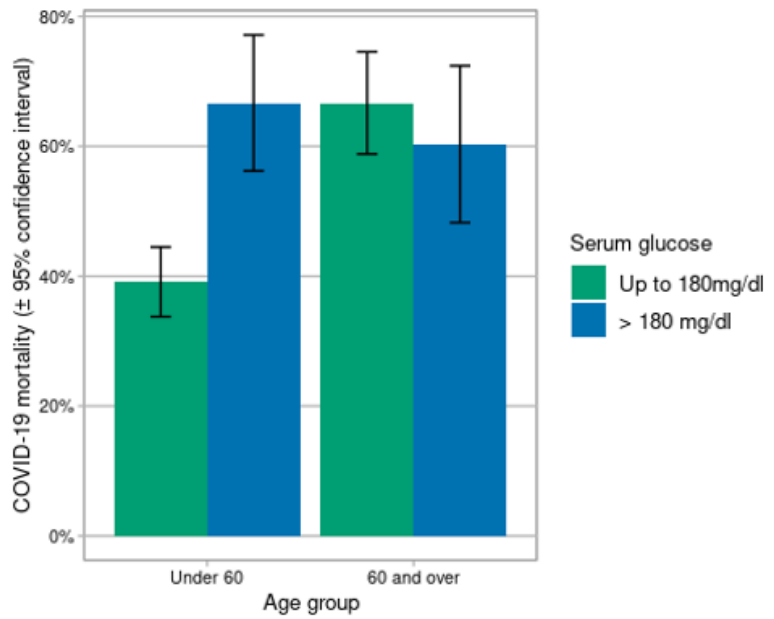

Figure 2: COVID-19 mortality according to hyperglycemia and age group. Confidence intervals for each of the time points was calculated assuming a binomial distribution. 


\section{Multivariate Analysis}

In multivariate analysis that included admission date sex, age, oxygen saturation, hyperglycemia, lactic dehydrogenase and D-dimer all of the exposures except sex and D-dimer were associated with COVID- 19 mortality in the under 60 group, while only oxygen saturation and lactic dehydrogenase were found to be independent factors in the 60 and over group (Table 3). The different directions observed for hyperglycemia in univariate analysis, that does not show statistical significance in the 60 and over group, is also observed in the multivariate model. Logistic regression that included all subjects and a hyperglycemia* age group interaction term suggests that hyperglycemia behaves differently as a risk factor for COVID-19 mortality in younger as compared to elderly patients $(\mathrm{p}=0.026)$.

\section{Discussion}

In this sample of 646 hospitalized COVID-19 patients, despite including a higher proportion of young patients than in many series $[3,4]$ overall mortality was high. Still, mortality was highest in the older age group. The 60 and over group presented a higher frequency of comorbidities, however these were not associated with mortality. In multivariate logistic regression age, oxygen saturation and lactic dehydrogenase were associated with mortality in the 60 and over age group. Hyperglycemia, defined as admission glucose over $180 \mathrm{mg} / \mathrm{dl}$, was associated with mortality in the under 60 age group but not in the aged 60 and over. Another study reports an association between elevated glucose and COVID-19 mortality [13], although to our knowledge differences by age group haven't been reported. After replicating in the respiratory tract SARSCoV-2 may access systemic circulation [14] and affect pancreatic function. It is possible that a considerable glucose increase is a more specific marker of SARS-CoV-2 affection to the pancreas in the younger age group. Subjects 60 and over had a higher frequency of diabetes history and $25 \%$ of individuals in this group had serum glucose above $224 \mathrm{mg} / \mathrm{dl}$. This could indicate that this group had previous pancreatic dysfunction. Moreover, in our population more subjects are obese among the under 60 age group. A correlation of 0.81 has been reported between ACE2 pancreatic expression and BMI [15]. This suggests that in our study subjects, the younger group has a higher susceptibility to pancreatic invasion by SARSCoV-2 that could explain the $66.7 \%$ mortality these patients when hyperglycemic. This is a single center study that involves patients evaluated within a limited time period, which reduces the possibility of assessment bias. A possible limitation of this study is its retrospective nature, although as mentioned both biases in exposure and outcome assessment are unlikely. Another possible limitation is that sample size is smaller in the 60 and over age group. This might explain why we only observe association with admission date in subjects aged under 60 while other studies have observed decreased mortality even in subjects 60 and over [12]. In the case of hyperglycemia, decreased power is an unlikely explanation, as there evidence of statistical interaction in an unstratified analysis.

\section{Conclusion}

In conclusion, in our population with a high prevalence of diabetes and obesity, hyperglycemia is a COVID-19 mortality predictor only in subjects aged under 60 .

\section{References}

1. (2020) World Health Organization. WHO Coronavirus Disease (COVID-19) Dashboard | WHO Coronavirus Disease (COVID-19)

2. Datos y Recursos (2020) Información referente a casos COVID-19 en México.

3. Figliozzi S, Masci PG, Ahmadi N, Tondi L, Koutli E, et al. (2020) Predictors of adverse prognosis in COVID-19: A systematic review and metaanalysis. Eur J Clin Invest 50(10): 13362.

4. Grasselli G, Zangrillo A, Zanella A, Antonelli M, Cabrini L, et al. (2020) Baseline Characteristics and Outcomes of 1591 Patients Infected with SARS-CoV-2 Admitted to ICUs of the Lombardy Region, Italy. JAMA -J Am Med Assoc 323(16): 1574-1581.

5. Polverino F, Stern DA, Ruocco G, Balestro E, Bassetti M, et al. (2020) Comorbidities, Cardiovascular Therapies, and COVID-19 Mortality: A Nationwide, Italian Observational Study (ItaliCO). Front Cardiovasc Med 9:7.

6. Basto Abreu A, Barrientos Gutiérrez T, Rojas Martínez R, Aguilar Salinas CA, López Olmedo N, et al. (2020) Prevalence of diabetes and lack of glycemic control in Mexico: Results of the 2016 Trial. Salud Publica Mex: 62(1): 50-59.

7. Liu K, Chen Y, Lin R, Han K (2020) Clinical features of COVID-19 in elderly patients: A comparison with young and middle-aged patients. Journal of Infection. WB Saunders Ltd. 80(6): 14-18.

8. Song J, Hu W, Yu Y, Shen X, Wang Y, et al. (2020) A comparison of clinical characteristics and outcomes in elderly and younger patients with covid-19. Med Sci Monit 26: 925047.

9. Becerra Muñoz VM, Núñez-Gil IJ, Eid CM, Aguado MG, Romero R, et al. (2020) Clinical profile and predictors of in-hospital mortality among older patients admitted for COVID-19. Age Ageing.

10. Sanchez Conejo AR, Gasca GA, López Jacinto EA, Zavala Sánchez HM, Mayeya López M, et al. (2020) Hospital reconversion due to the pandemic. In: Acosta Altamirano G, Camacho Olivares G, Carrasco Valdéz M, Robledo Cayetano M, (Eds). HRAEI strategies in the face of the COVID-19 challenge. First. Ixtapaluca 30-39.

11. Moghissi ES, Korytkowski MT, Dinardo M, Einhorn D, Hellman R, et al. (2009) American association of clinical endocrinologists and american diabetes association consensus statement on inpatient glycemic control. Endocrine Practice. Endocr Pract 15: (353-369).

12. Horwitz LI, Jones SA, Cerfolio RJ, Francois F, Greco J, et al. (2020) Trends in COVID-19 Risk-Adjusted Mortality Rates. J Hosp Med.

13. Bode B, Garrett V, Messler J, McFarland R, Crowe J, et al. (2020) Glycemic Characteristics and Clinical Outcomes of COVID-19 Patients Hospitalized in the United States. J Diabetes Sci Technol 14(4): 813-821.

14. Torres Estrella CU, Sierra Martínez M, Chávez Martínez MÁ, García Salazar E, Acosta Altamirano G (2020) Plasma Exchange as a Therapeutic Alternative to Eliminate Viral RNA and Macromolecules Involved in the Immune Response of Patients with Sars-Cov-2. Biomed J Sci Tech Res 29(1).

15. Kusmartseva I, Wu W, Syed F, Van Der Heide V, Jorgensen M, et al. (2020) Expression of SARS-CoV-2 Entry Factors in the Pancreas of Normal Organ Donors and Individuals with COVID-19. Cell Metab. 